\title{
A Co(II)-Based Metallo-Supramolecular Polymer as a Novel Enzyme Immobilization Matrix for Electrochemical Glucose Biosensing
}

Chih-Yu Hsu, ${ }^{a, b}$ Takashi Sato, ${ }^{a, b}$ Satoshi Moriyama, ${ }^{b, c}$ and Masayoshi Higuchi ${ }^{*, a, b}$

${ }^{a}$ Electronic Functional Materials Group, Polymer Materials Unit, National Institute for Materials Science (NIMS), 1-1 Namiki, Tsukuba 305-0044, Japan

${ }^{\mathrm{b}}$ CREST Project, Japan Science and Technology Agency (JST-CREST), Tokyo, 102-0076, Japan

${ }^{\mathrm{c}}$ International Center for Materials Nanoarchitectonics (MANA), NIMS, 1-1 Namiki, Tsukuba 305-0044, Japan

*Corresponding author. E-mail: higuchi.masayoshi@nims.go.jp; tel.: +81-29-860-4744; fax:

$+81-29-860-4721$. 


\begin{abstract}
$\operatorname{ABSTRACT}^{1}$
A Co(II)-based metallo-supramolecular polymer (polyCo) was used as a novel matrix to immobilize glucose oxidase (GOx) for enzymatic glucose sensing. A facile fabrication of a modified glassy carbon electrode (GCE) was achievable by a one-step co-immobilization method. UV-vis spectroscopy and cyclic voltammetry were used to characterize the electrochemical biosensor and the sensing mechanism of the reaction on the polyCo-modified GCE. After the optimization of composition and loading of the polyCo/GOx GCE, the fabricated sensor demonstrated excellent amperometric quantification of glucose, with high sensitivity $(47.1 \mu \mathrm{A}$ $\mathrm{mM}^{-1} \mathrm{~cm}^{-2}$ ) in the linear range of up to $1.4 \mathrm{mM}$. The selectivity, based on the enzyme matrix without any pretreatment, was also sufficiently high. The outstanding stability of the sensor supported our assumption that metallo-supramolecular polymers can be used as electrode materials for glucose biosensors.
\end{abstract}

Keywords: biosensing, enzyme, glucose, metallo-supramolecular polymer

${ }^{1} \mathrm{Co}(\mathrm{II})$-based metallo-supramolecular polymer, polyCo; glucose oxidase, GOx; glassy carbon electrode, GCE; tetrathiafulvalene-tetracyanoquinodimethane, TTF--TCNQ; cyclic voltammetry, CV 


\section{Introduction}

Glucose biosensors have attracted significant interest, owing to their importance for several medical conditions, particularly diabetes mellitus, which generally affects people in developed societies [1]. This endocrine disorder of carbohydrate metabolism may cause life-threatening complications if the concentration of glucose in blood deviates from its normal range of 80-120 $\mathrm{mg} \mathrm{dL}^{-1}[2]$. A considerable demand from millions of diabetics accounts for the fact that glucose biosensors maintain an approximately $85 \%$ share of the biosensor market and continuously drives evolutionary research since 1962 [2-4]. Most of the abovementioned studies have focused on enzymatic and amperometric methodologies for specific and facile glucose sensing in the socalled second-generation glucose biosensors [5]. The flavin adenine dinucleotide (FAD) redox center in the glucose oxidase (GOx) enzyme can perform electron transfer to the modified electrode [6]. Several electrode materials were employed to overcome the electron tunneling between the enzyme and the electrode surface, such as organic conducting charge-transfer complexes, conducting polymers, and nanomaterials $[2,3]$. Nevertheless, the bioactivity of GOx can be altered by enzyme denaturation during glucose detection and still requires careful investigation $[7,8]$. The possible conformational change of GOx is attributed to the barrier-like protein shell that hinders FAD from effortless electron transfer to the electrode. Consequently, several effective mediators for GOx were introduced for the second generation biosensors, e.g.

ferrocenes, tetrathiafulvalene-tetracyanoquinodimethane (TTF--TCNQ), quinones, and phenothazine/phenoxazine compounds [3]. Most of the mediators were originally diffusionbased, and there were also efforts to immobilize the mediators on the electrode film for the stabilization of the biosensors $[9,10]$. However, the mediating efficiency may decrease after 
immobilization on the electrode. Therefore, keeping the balance between the enzymatic and electrochemical activities of GOx is vital for the second-generation glucose biosensors.

Metallo-supramolecular polymers, i.e. metal ions linked with bridging organic ligands via non-covalent interactions, are a new class of hybrid materials, which have attracted a growing interest in the past decade, due to their tunable characteristics $[11,12]$. The motif structures, thermodynamic stabilities, and dynamic properties of these materials can be adjusted depending on the integration of the organic and inorganic components on the atomic scale. Advanced functionalities are endowed with greater structural complexities and interactions, compared with the respective isolated components. These advantages are visualized by the expansion of the corresponding applications [13-15]. Specifically for bio-applications, the organic ligands can be used as efficient matrixes to immobilize bio-recognition molecules with metallic centers as good electronic signal conductors. Based on this concept, similar materials, such as redox polymers $[10,16,17]$, supramolecular complexes [18], and metalloorganic coordination polymers $[19,20]$ have been applied in glucose biosensors.

In the present work, the application of a Co(II)-bis(terpyridine)-based metallo-supramolecular polymer (polyCo) in an amperometric glucose biosensor was investigated. The modified electrodes can be easily fabricated by co-immobilizing GOx with the water-soluble polymer (Scheme 1). The metal-ligand architecture provides unique properties for enzymatic sensing, such as effective immobilization and a highly efficient electron transfer based on the metal-toligand charge transfer (MLCT), intervalence charge transfer (IVCT), and intra-ligand transitions. It also enables electron transfer between GOx and the polymer-modified electrode. In addition, our proposed polymer is supposed to tackle the problem of GOx denaturation caused by the 
electron transfer process with the aid of the electrocatalytic cobalt ion, allowing the fabrication of an efficient and stable glucose biosensor.

\section{Experimental}

\subsection{Chemicals}

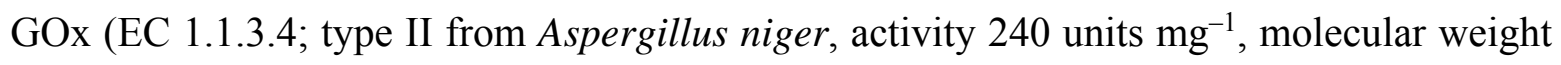
(Mw) 186000), $\mathrm{D}(+)$-glucose $\left(\geq 98 \%\right.$ ), and cobalt(II) acetate $\left(\mathrm{Co}(\mathrm{OAc})_{2}\right)$ were purchased from Wako Chemicals. Phosphate buffer saline tablets were purchased from Sigma. 4',4"'-(1,4phenylene)bis(2,2':6',2"-terpyridine) (97\%), ascorbic acid ( $\geq 99 \%$ ), and uric acid ( $\geq 99 \%)$ were purchased from Aldrich. All chemicals were of analytical grade or better quality and were used as received. All solutions were prepared with ultrapure water throughout the experiments, with the exception of methanol (Wako Chemicals), which was dehydrated and then used in the polymer synthesis. The phosphate buffer solution (PBS) was prepared from the above tablets to obtain a $0.01 \mathrm{M}$ phosphate buffer containing $0.0027 \mathrm{M} \mathrm{KCl}$ and $0.137 \mathrm{M} \mathrm{NaCl}$ (pH 7.4). Prior to the measurements, a glucose solution was prepared and stored at room temperature for $24 \mathrm{~h}$, to allow for mutarotation due to the interconversion of $\alpha$ and $\beta$ anomeric forms.

\subsection{Apparatus}

All electrochemical experiments, including cyclic voltammetry (CV) and amperometric measurements, were carried out on an ALS/CHI Model 612B electrochemical workstation (CH Instruments, Inc.). A conventional three-electrode system was used with a bare/modified glassy carbon electrode (GCE, $3 \mathrm{~mm}$ diameter, BAS Inc., Japan) as the working electrode, a platinum 
wire as the counter electrode, and $\mathrm{Ag} / \mathrm{AgCl} / \mathrm{saturated} \mathrm{KCl}$ (BAS Inc., Japan) as the reference electrode. All potentials reported are relative to the $\mathrm{Ag} / \mathrm{AgCl} / \mathrm{sat}$. $\mathrm{KCl}$ reference electrode. $\mathrm{UV}$ vis spectra were recorded by a Shimadzu UV-2550 UV-visible spectrophotometer. Fluorescence spectra were acquired on a Shimadzu RF-5300PC spectrofluorophotometer by excitation of the GOx tryptophan (Trp) at $290 \mathrm{~nm}$ and that of FAD at $467 \mathrm{~nm}$. All experiments were performed at room temperature.

\subsection{Polymer Synthesis}

PolyCo was synthesized according to our previously reported procedure [21]. An equimolar amount of 4',4"'-(1,4-phenylene)bis(2,2':6',2"-terpyridine) and $\mathrm{Co}(\mathrm{OAc})_{2}$ was refluxed in $\mathrm{MeOH}$ for $24 \mathrm{~h}$ under argon. The resulting solution was cooled to room temperature and filtered to remove a small amount of insoluble residue. The filtrate was slowly evaporated to remove the solvent, and the product was collected and further dried in vacuum overnight to give polyCo ( $>$ $90 \%$ ). In order to compare the unit sensing capability based on the metallic center of Co ion and the GOx enzyme, here we define the unit molar concentration (unit M) for the polymer. The unit $\mathrm{M}$ of polyCo is calculated based on the molecular weight of the repeated unit of polyCo (scheme 1) including the metallic center, ligands, and counter ion to be $717 \mathrm{~g} / \mathrm{mol} .0 .7$ unit $\mathrm{mM}$, i.e. 0.5 $\mathrm{mg} \mathrm{mL} \mathrm{m}^{-1}$, of the polymer was dissolved in water before use.

\subsection{Preparation of the Enzyme-Functionalized Electrodes}

Prior to surface modification, the GCE was polished with alumina slurry $(0.05 \mu \mathrm{m}$ particle size) and sequentially sonicated in water and ethanol for five minutes. After drying, $5 \mu \mathrm{L}$ of the above polyCo solution or of a mixed solution containing 0.7 unit $\mathrm{mM}$ polyCo and $0.05 \mathrm{mM} \mathrm{GOx}$ (unless otherwise stated) were cast on the well-cleaned GCE, which was then dried at room 
temperature. Subsequently, the prepared polyCo/GCE or polyCo/GOx/GCE enzyme-modified electrodes were stored at $4{ }^{\circ} \mathrm{C}$ when not in use. It is notable that at high concentration of the casting solution the polymers prefer to aggregate, due to the strong ionic interaction between metallo-polymer chains. Once the polymer film is formed, it shows high stability towards water, and harsh conditions, such as sonication, are required to dissolve the film.

\section{Results and discussion}

\subsection{Electrochemical glucose sensing mechanism}

In the enzymatic sensing approach, a mixed aqueous solution of GOx and polyCo was used for the electrode modification. UV-vis spectroscopy measurements confirmed the conformational change of the enzyme, i.e. the toxicity of polyCo to GOx. The absorbance spectra of the solutions of polyCo, GOx, and their mixture are shown in Fig. 1. The spectrum of polyCo displays a pronounced $\pi-\pi^{*}$ transition around $300 \mathrm{~nm}$ and a weak $d-d^{*}$ transition of the cobalt ion at ca. $520 \mathrm{~nm}$. For pure GOx, the absorption peak at ca. $277 \mathrm{~nm}$ is characteristic of polypeptide chains, and the other two peaks at ca. 381 and $455 \mathrm{~nm}$ are characteristic of the oxidized form of the flavin group [22]. The spectrum of the GOx/polyCo mixture shows three pronounced absorption bands. Since the peaks corresponding to pure GOx retained their position and shape in the spectrum of the mixture, the intactness of the enzyme bio-structure and absence of conformational change were confirmed.

Fig. 2 shows the electrochemical responses of the enzyme-modified electrodes, GOx/GCE, polyCo/GCE, and polyCo/GOx/GCE, which were characterized by cyclic voltammetry $(\mathrm{CV})$ in 
phosphate buffer solution (PBS). No redox peaks were observed for the GCE coated with a GOx film, indicating absence of electron transfer processes on this modified electrode (curve 1). For the polyCo/GCE electrode, a reversible redox couple was detected at a formal potential $\left(E^{\circ}\right)$ of ca. $-0.86 \mathrm{~V}$ by $\mathrm{CV}$ in aqueous PBS (Fig. S1), attributable to electron transfer at the $\mathrm{Co}(\mathrm{II}) / \mathrm{Co}(\mathrm{I})$ metal center [23-25]. On the contrary, polyCo/GOx/GCE displayed a reduction peak at ca. -0.59 $\mathrm{V}$ in addition to the redox couple of polyCo (curve 2 in Fig. 2). This quasi-reversible peak is a result of electron transfer between the FAD redox center in GOx and the modified electrode surface [26]. The glucose sensing response of the enzyme-modified electrodes is shown in Fig. 2. When air-saturated PBS (curve 2) was used instead of deoxygenated PBS (curve 3), the reduction peak currents for both GOx and polyCo showed a significant increase. A two-proton, two-electron transfer from the modified electrode to the enzyme FAD moiety was proposed [27]:

$$
\begin{aligned}
& \mathrm{GOx}(\mathrm{FAD})+2 \mathrm{e}^{-}+2 \mathrm{H}^{+} \leftrightharpoons \mathrm{GOx}\left(\mathrm{FADH}_{2}\right) \\
& \mathrm{GOx}\left(\mathrm{FADH}_{2}\right)+\mathrm{O}_{2} \rightarrow \mathrm{GOx}(\mathrm{FAD})+\mathrm{H}_{2} \mathrm{O}_{2}
\end{aligned}
$$

In the presence of oxygen, the oxidized FAD form was regenerated (eq. (2)), and thus the reduction peak current of GOx at ca. $-0.59 \mathrm{~V}$ increased (eq. (1)). In addition, we proposed the existence of an electron transfer interaction between GOx and polyCo as follows:

$$
\begin{aligned}
& \text { polyCo }^{2+}+\mathrm{e}^{-} \leftrightharpoons \text { polyCo }^{+} \\
& \mathrm{GOx}(\mathrm{FAD})+2 \mathrm{polyCo}^{+}+2 \mathrm{H}^{+} \rightarrow \mathrm{GOx}\left(\mathrm{FADH}_{2}\right)+2 \mathrm{polyCo}^{2+}
\end{aligned}
$$

The reduced polyCo ${ }^{+}$was re-oxidized by $\mathrm{GOx}(\mathrm{FAD})$ in the presence of oxygen (eq. (4)). Consequently, the reduction peak current of polyCo at ca. $-0.86 \mathrm{~V}$ increased. 
In the presence of glucose (curve 4), the reduction peak current of GOx went down to essentially zero, and that of polyCo showed a significant decrease. The consumption of GOx in the enzymatic reaction with glucose greatly reduced its concentration:

$$
\text { Glucose }+\mathrm{GOx}(\mathrm{FAD}) \rightarrow \text { gluconolactone }+\mathrm{GOx}\left(\mathrm{FADH}_{2}\right)
$$

Since the oxidized form of FAD was consumed, less of the oxidized polyCo ${ }^{2+}$ was regenerated, leading to a decrease of the polyCo reduction peak current.

\subsection{Biosensor performance}

According to the stepwise nature of electrocatalytic processes, the loading amounts of GOx and polyCo on GCE influence the biosensor performance. It is noteworthy that at low concentrations of polyCo, GOx was weakly immobilized, playing a minor role in signal transduction (Fig. S2(a)). On the contrary, excess polyCo resulted in no sensing response to glucose (Fig. S2(b)), owing to an excessive coverage of GOx, which hinders glucose mass transport. The concentration of polyCo in the casting solution was varied in the range of $0.3-1.4$ unit $\mathrm{mM}$, with GOx fixed at $0.05 \mathrm{mM}$. The sensing response of the resulting modified electrodes was investigated by linear sweep voltammetry (LSV) in the presence of glucose (Fig. 3(a)), summarized in Fig. 3(b). The response reached a maximum at a polyCo concentration of ca. 0.7 unit $\mathrm{mM}$, indicating the optimal condition to immobilize GOx while maintaining sufficient matrix permeability and mediating ability. Fig. 3(c) and 3(d) show the dependence of the sensing response on the GOx loading, varied from 0.005 to $0.1 \mathrm{mM}$. The response gradually reached its maximum at around $0.05 \mathrm{mM}$ and then decreased. Excess GOx adversely affected the electron transfer in the system, forming an insulating protein layer and, therefore, reducing the sensing response. This can be elucidated from the LSV peak potentials of the enzyme-modified 
electrodes. As the GOx loading increases, the potential bias required for driving the electrochemical reaction of either GOx or polyCo becomes larger, i.e. negatively shifted. This behavior was not observed when solely the polyCo loading was varied. Consequently, the solution containing 0.7 unit $\mathrm{mM}$ polyCo and $0.05 \mathrm{mM}$ GOx was determined to be optimal for the biosensor preparation.

For the subsequent amperometric detection, a suitable sensing potential was determined to be equal to $-0.8 \mathrm{~V}$, with a current plateau observed according to the net current response from the LSV, scanned at a rate of $1 \mathrm{mV} \mathrm{s}^{-1}$ (Fig. S3). Fig. 4 shows the current responses for polyCo/GOx/GCE upon successive addition of glucose to the PBS electrolyte. The reduction currents reached $95 \%$ of their steady states around 5-20 s after each addition, exhibiting a rapid analytical response. A calibration curve for the average steady-state reduction current density as a function of glucose concentration was plotted, shown in the inset of Fig. 4. The above relationship was linear in the glucose concentration range of 0.1 to $1.4 \mathrm{mM}$, with a correlation coefficient $\left(\mathrm{R}^{2}\right)$ of 0.999 . The sensitivity was determined to be $47.1 \mu \mathrm{A} \mathrm{mM}{ }^{-1} \mathrm{~cm}^{-2}$ from the linear regression slope. The limit of detection (LOD) for the biosensor was estimated to be 82.8 $\mu \mathrm{M}(\mathrm{S} / \mathrm{N}=3)$ from the amperometric background current, i.e. the current recorded in absence of glucose. The reports of glucose sensing using a GOx-immobilized cobalt supramolecular polymer modified electrode are very rare [28-30]. All of these investigations employed cobaltporphyrin based supramolecular materials as redox mediators, with the sensing principle based on oxygen detection, i.e. the principle used in first generation glucose biosensors. To the best of our knowledge, using a cobalt-supramolecular polymer as an enzyme-immobilizing matrix for an amperometric glucose biosensor was not reported. 
At concentrations higher than $1.4 \mathrm{mM}$ the response slowly reached saturation. This asymptotic electrocatalytic behavior is described by the Michaelis-Menten (MM) mechanism, where an intermediate complex forms between GOx and glucose, followed by its decomposition:

$$
\mathrm{GOx}(\mathrm{FAD})+\text { Glucose } \underset{k_{-1}}{\stackrel{k_{1}}{\longrightarrow}}[\mathrm{GOx}(\mathrm{FAD}) \cdots \text { Glucose }] \stackrel{k_{c}}{\longrightarrow} \mathrm{GOx}\left(\mathrm{FADH}_{2}\right)+\text { gluconolactone }
$$

where $K_{\mathrm{M}}=\left(k_{\mathrm{c}}+k_{-1}\right) / k_{1}$ is the $\mathrm{MM}$ constant $\left(\mathrm{mol} \mathrm{cm}{ }^{-3}\right)$, and $k_{\mathrm{c}}$ is the complex decomposition rate constant $\left(\mathrm{s}^{-1}\right)$. The $\mathrm{MM}$ equation for the above mechanism can be written as:

$$
i_{\mathrm{CAT}}=\frac{n_{1} F A k_{\mathrm{c}} c_{\text {glucose }} \Gamma}{K_{\mathrm{M}}+c_{\text {glucose }}}
$$

where $i_{\mathrm{CAT}}$ is the catalytic current, $n_{1}$ is the number of electrons involved in the overall catalytic reaction $\left(n_{1}=1\right)$, and all other symbols have their usual meanings. The above equation can also be re-written as:

$$
i_{C A T}=\frac{i_{M} c_{\text {glucose }}}{K_{\mathrm{M}}+c_{\text {glucose }}}
$$

where $i_{M}$ is the maximum catalytic current, given by $\boldsymbol{i}_{\mathbf{M}}=n_{1} F A k_{\mathrm{c}} \boldsymbol{\Gamma}$. The $K_{\mathrm{M}}$ value can be obtained using the Lineweaver-Burk transformation of the MM equation [31,32]:

$\frac{1}{i_{\text {CAT }}}=\frac{K_{\mathrm{M}}}{i_{\mathrm{M}} c_{\text {glucose }}}+\frac{1}{i_{\mathrm{M}}}$

An average value of $0.69 \mathrm{mM}$ was calculated for $K_{\mathrm{M}}$ by these three approaches. This low $K_{\mathrm{M}}$ value implies that mixing with polyCo preserves the native GOx structure leading to high affinity and activity towards glucose [26]. 


\subsection{Interference and stability study}

For a practically useful glucose biosensor, the suppression of interference from the endogenous species in human blood, such as ascorbic acid (AA) and uric acid (UA), is of great importance. The normal physiological levels of glucose, AA and UA are 3-8, 0.1 and $0.1 \mathrm{mM}$, respectively [33]. Therefore, the concentration of AA and UA for the selectivity evaluation (0.04 $\mathrm{mM}, c f .0 .4 \mathrm{mM}$ glucose) was selected to be an order of magnitude lower than that of glucose. The amperometric response of the non-pretreated polyCo/GOx/GCE to $0.04 \mathrm{mM}$ AA and UA was rather small compared to that observed for $0.4 \mathrm{mM}$ glucose (Fig. 5). In addition, a stable response was still observed for each addition of glucose, despite the two interfering species were added in between, showing reliable selectivity of the biosensor.

The reproducibility and stability of the biosensor response were examined by amperometric detection of $0.4 \mathrm{mM}$ glucose in PBS. The responses of five independently prepared polyCo/GOx/GCEs exhibited satisfactory reproducibility with an RSD of $8.1 \%$ (Fig. S4). As for the stability, the prepared modified electrode was stored at $4{ }^{\circ} \mathrm{C}$, being exposed to room temperature only when a measurement was carried out (once a week). Three successive measurements were performed week by week, as shown in Fig. S5, with the current density changes summarized in Table 1. The data analysis gave an average RSD of $13.5 \%$ based on each week, a value that confirms repeatable and reliable sensing. After four-week storage, the sensitivity of the biosensor still remained at $98.7 \%$ of its original value. The highly stable sensor performance proves that the enzymatic nature of GOx as well as its electrochemical activity can be preserved in the polyCo matrix. 
The investigation of GOx denaturation by fluorescence spectroscopy provided more direct evidence on the conformational stability of the GOx structure. $\lambda_{\max }$ of the GOx tryptophan (Trp) fluorescence $\left(\lambda_{\text {exc }}\right.$ at $290 \mathrm{~nm}$ ) was used to monitor the GOx unfolding, with the FAD fluorescence intensity $\left(\lambda_{\text {exc }}\right.$ at $467 \mathrm{~nm}$ ) increasing when the protein is unfolded and provides greater exposure [34]. Fig. 6 shows the fluorescence change of the enzyme thin-film electrode after a 100-fold repetition of the sensing, with only a slight shift of the $\operatorname{Trp} \lambda_{\max }$ (362 to $366 \mathrm{~nm}$ ) and a slight increase of the FAD intensity detected, which indicates negligible GOx denaturation. The results prove that polyCo is an efficient and reliable matrix for GOx immobilization for a stable amperometric glucose biosensor.

\section{Conclusions}

It was demonstrated that a $\mathrm{Co}(\mathrm{II})$-bis(terpyridine)-based metallo-supramolecular polymer (polyCo) can be used to construct an enzyme-modified electrode by a simple one-step coimmobilization with GOx. The newly developed electrode exhibited excellent electrocatalytic ability for glucose detection, being a good candidate for a novel second generation glucose biosensor. The optimized modified electrode showed excellent amperometric sensing performance, with the linear range, sensitivity, response time, and LOD of $0.1-1.4 \mathrm{mM}$ (correlation coefficient $\mathrm{R}^{2}=0.999$ ), $47.1 \mu \mathrm{A} \mathrm{mM}^{-1} \mathrm{~cm}^{-2}, 5-20 \mathrm{~s}$, and $82.8 \mu \mathrm{M}$, respectively. The MM kinetic mechanism was observed at concentrations outside of the linear range, and the apparent $\mathrm{MM}$ constant $\left(K_{\mathrm{M}}\right)$ was estimated to equal $0.69 \mathrm{mM}$. Interference of AA and UA with the glucose detection was shown to be negligible, without the need of any pretreatment for polyCo/GOx/CGE. Besides, fluorescence spectroscopy investigation of the GOx structure 
together with the outstanding stability of the biosensor showed that the utilization of polyCo solves the problem of GOx denaturation in glucose biosensors. These characteristics proved polyCo to be an applicable electrode material for glucose monitoring. Metallo-supramolecular polymers with properties tunable by modifying the ligands or metallic centers have great potential for the development of glucose biosensors.

\section{Acknowledgment}

This work was supported by the CREST Project, Japan Science and Technology Agency (JST). 


\section{References}

1. J. E. Shaw, R. A. Sicree, P. Z. Zimmet, Global estimates of the prevalence of diabetes for 2010 and 2030, Diabetes Res. Clin. Pract 87 (2010) 4-14.

2. J. Wang, Electrochemical glucose biosensors, Chem. Rev. 108 (2008) 814-825.

3. C. Chen, Q. Xie, D. Yang, H. Xiao, Y. Fu, Y. Tan, S. Yao, Recent advances in electrochemical glucose biosensors: a review, RSC adv. 3 (2013) 4473-4491.

4. L. C. Clark Jr., C. Lyons, Electrode systems for continuous monitoring in cardiovascular surgery, Ann. N. Y. Acad. Sci. 102 (1962) 29-45.

5. J. Wang, Glucose biosensors: 40 years of advances and challenges, Electroanalysis 13 (2001) 983-988.

6. A. L. Ghindilis, P. Atanasov, E. Wilkins, Enzyme-catalyzed direct electron transfer: Fundamentals and analytical applications, Electroanalysis 9 (1997) 661-674.

7. Y. Su, Q. Xie, C. Chen, Q. Zhang, M. Ma, S. Yao, Electrochemical quartz crystal microbalance studies on enzymatic specific activity and direct electrochemistry of immobilized glucose oxidase in the presence of sodium dodecyl benzene sulfonate and multiwalled carbon nanotubes, Biotechnol. Prog. 24 (2008) 262-272.

8. Y. Wang, Y. Yao, Direct electron transfer of glucose oxidase promoted by carbon nanotubes is without value in certain mediator-free applications, Microchim. Acta 176 (2012) 271-277. 
9. P. D. Hale, T. Inagaki, H. I. Karan, Y. Okamoto, T. A. Skotheim, A new class of amperometric biosensor incorporating a polymeric electron-transfer mediator, J. Am. Chem. Soc. 111 (1989) 3482-3484.

10. L. Deng, Y. Liu, G. Yang, L. Shang, D. Wen, F. Wang, Z. Xu, S. Dong, Molecular "wiring" glucose oxidase in supramolecular architecture, Biomacromolecules 8 (2007) 2063-2071.

11. F. Pichot, J. H. Beck, C. M. Elliott, A series of multicolor electrochromic ruthenium(II) trisbipyridine complexes: synthesis and electrochemistry, J. Phys. Chem. A 103 (1999) $6263-6267$.

12. H. Nishihara, K. Kanaizuka, Y. Nishimori, Y. Yamanoi, Construction of redox- and photofunctional molecular systems on electrode surface for application to molecular devices, Coord. Chem. Rev. 251 (2007) 2674-2687.

13. R. Shunmugam, G. N. Tew, Side chain terpyridine motifs for supramolecular materials, J. Am. Chem. Soc. 127 (2005) 13567-13572.

14. J. R. Kumpfer, S. J. Rowan, Thermo-, photo-, and chemo-responsive shape-memory properties from photo-cross-linked metallo-supramolecular polymers, J. Am. Chem. Soc. 133 (2011) 12866-12874.

15. F. S. Han, M. Higuchi, D. G. Kurth, Metallo-supramolecular polymers based on functionalized bis-terpyridines as novel electrochromic materials, Adv. Mater. 19 (2007) 3928-3931. 
16. E. J. Calvo, A. Wolosiuk, Supramolecular architectures of electrostatic self-assembled glucose oxidase enzyme electrodes, ChemPhysChem 5 (2004) 235-239.

17. H. Deng, A. K. L. Teo, Z. Gao, An interference-free glucose biosensor based on a novel low potential redox polymer mediator, Sens. Actuator B-Chem. 191 (2014) 522-528.

18. W. A. Alves, P. A.; Fiorito, S. I. C. de Torresi, R. M. Torresi, Design of molecular wires based on supramolecular structures for application in glucose biosensors, Biosens. Bioelectron. 22 (2006) 298-305.

19. Y. Fu, P. Li, L. Bu, T. Wang, Q. Xie, J. Chen, S. Yao, Exploiting metal-organic coordination polymers as highly efficient immobilization matrixes of enzymes for sensitive electrochemical biosensing, Anal. Chem. 83 (2011) 6511-6517.

20. Y. Guo, Y. Han, S. Shuang, C. Dong, Rational synthesis of graphene-metal coordination polymer composite nanosheet as enhanced materials for electrochemical biosensing, J. Mater. Chem. 22 (2012) 13166-13173.

21. F. S. Han, M. Higuchi, D. G. Kurth, Metallosupramolecular polyelectrolytes self-assembled from various pyridine ring-substituted bisterpyridines and metal ions: photophysical, electrochemical, and electrochromic properties, J. Am. Chem. Soc. 130 (2008) 2073-2081.

22. J. Li, Y. Tang, J. Yang, Z. Yang, Y. Zhang, X. Hu, Cage-like PbS nanostructure for the construction of novel glucose electrochemical biosensor, Sens. Actuator B-Chem. 190 (2014) $549-554$. 
23. O. Buriez, E. Labbé, J. Périchon, Unexpected stabilization of a simple cobalt(I) salt in acetonitrile at a glassy carbon electrode, J. Electroanal. Chem. 593 (2006) 99-104.

24. K. T. Potts, D. A. Usifer, A. Guadalupe, H. D. Abruna, 4-Vinyl-, 6-vinyl-, and 4'-viny1$2,2^{\prime}: 6^{\prime}, 2^{\prime \prime}$-terpyridinyl ligands: their synthesis and the electrochemistry of their transitionmetal coordination complexes, J. Am. Chem. Soc. 109 (1987) 3961-3967.

25. D. Chen, P.-L. Fabre, O. Reynes, Electrocarboxylation of chloroacetonitrile by a Cobalt(I) complex of terpyridine, Electrochim. Acta 56 (2011) 8603-8610.

26. Y. Yu, Z. Chen, S. He, B. Zhang, X. Li, M. Yao, Direct electron transfer of glucose oxidase and biosensing for glucose based on PDDA-capped gold nanoparticle modified graphene/multi-walled carbon nanotubes electrode, Biosens. Bioelectron. 52 (2014) 147152.

27. A. Haouz, C. Twist, C. Zentz, P. Tauc, B. Alpert, Dynamic and structural properties of glucose oxidase enzyme, Eur. Biophys. J. 27 (1998) 19-25.

28. K. I. Ozoemena, T. Nyokong, Novel amperometric glucose biosensor based on an etherlinked cobalt(II) phthalocyanine-cobalt(II) tetraphenylporphyrin pentamer as a redox mediator, Electrochim. Acta 51 (2006) 5131-5136.

29. D. S. Kumar, K. Nakamura, A. Shoji, Y. Yoshida, Cobalt tetraphenylporphine (CoTPP) film as glucose and hydrogen peroxide sensors after immobilizing glucose oxidase (GOx), Sens. Mater. 18 (2006) 339-352. 
30. W. Chen, Y. Ding, J. Akhigbe, C. Brückner, C. M. Li, Y. Lei, Enhanced electrochemical oxygen reduction-based glucose sensing using glucose oxidase on nanodendritic poly[mesotetrakis(2-thienyl)porphyrinato]cobalt(II)-SWNTs composite electrodes, Biosens. Bioelectron. 26 (2010) 504-510.

31. R. Thangamuthu, S. M. Senthil Kumar, K. Chandrasekara Pillia, Direct amperometric determination of 1-ascorbic acid (Vitamin C) at octacyanomolybdate-doped-poly(4vinylpyridine) modified electrode in fruit juice and pharmaceuticals, Sens. Actuator B-Chem. 120 (2007) 745-753.

32. D. L. Scott, E. F. Bowden, Enzyme-substrate kinetics of adsorbed cytochrome c peroxidase on pyrolytic graphite electrodes, Anal. Chem. 66 (1994) 1217-1223.

33. E. Scavetta, B. Ballarin, D. Tonelli, A cheap amperometric and optical sensor for glucose determination, Electroanalysis 22 (2010) 427-432.

34. E. Tellechea, K. J. Wilson, E. Bravo, K. Hamad-Schifferli, Engineering the interface between glucose oxidase and nanoparticles, Langmuir 28 (2012) 5190-5200. 
Scheme 1. Schematic illustration of the glucose biosensing electrode and the chemical structure of polyCo.
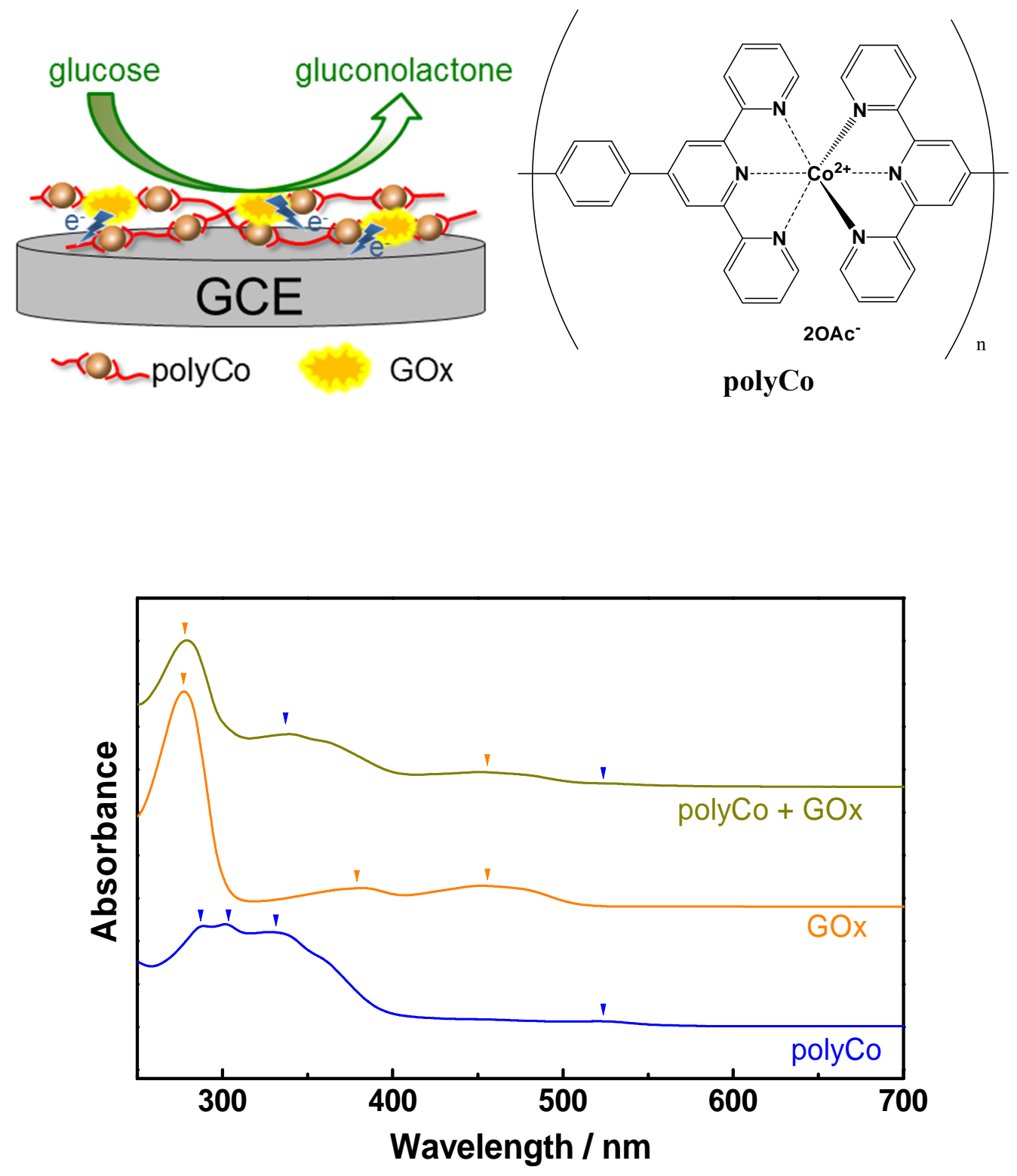

Fig. 1. UV-vis spectra of the solutions of polyCo, GOx, and their mixture. The colored markers are for easier comparison for the peaks from pure polyCo and GOx solutions. 


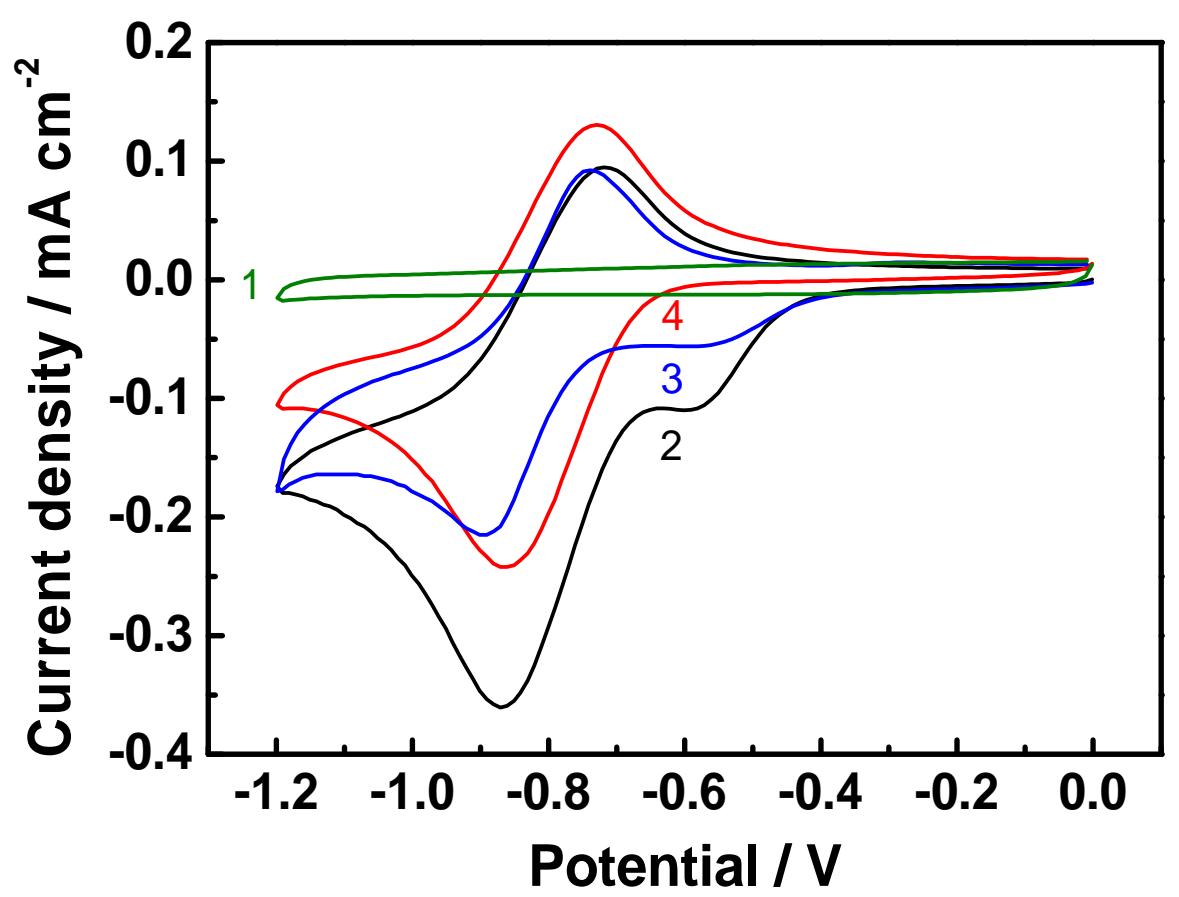

Fig. 2. $\mathrm{CVs}$ of $\mathrm{GOx} / \mathrm{GCE}$ in air-saturated $\mathrm{PBS}(1)$, polyCo/GOx/GCE in air-saturated $\mathrm{PBS}$ (2), nitrogen-saturated PBS (3), and $1 \mathrm{mM}$ glucose in air-saturated PBS (4) at a scan rate of $100 \mathrm{mV}$ $\mathrm{S}^{-1}$ 

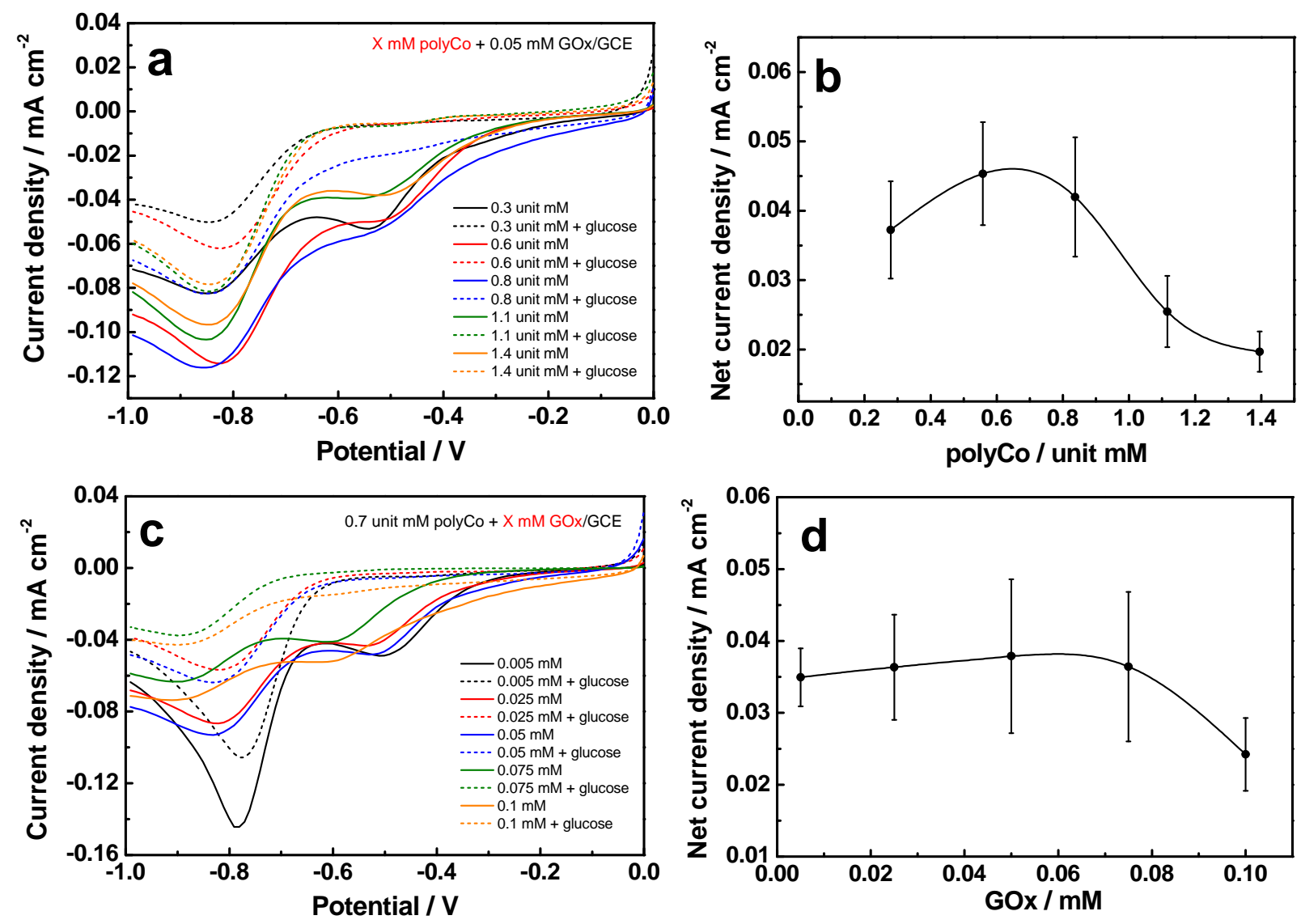

Fig. 3. Effect of the loading amount of polyCo $(a, b)$ and GOx $(c, d)$ on the LSV responses and the net current density of polyCo/GOx/GCE in absence/presence of $1 \mathrm{mM}$ glucose in $\mathrm{PBS}$ at a scan rate of $5 \mathrm{mV} \mathrm{s}^{-1}$. The net current density was obtained by subtracting the background current (without glucose) at $-0.8 \mathrm{~V}$ from the reaction current (with glucose). 


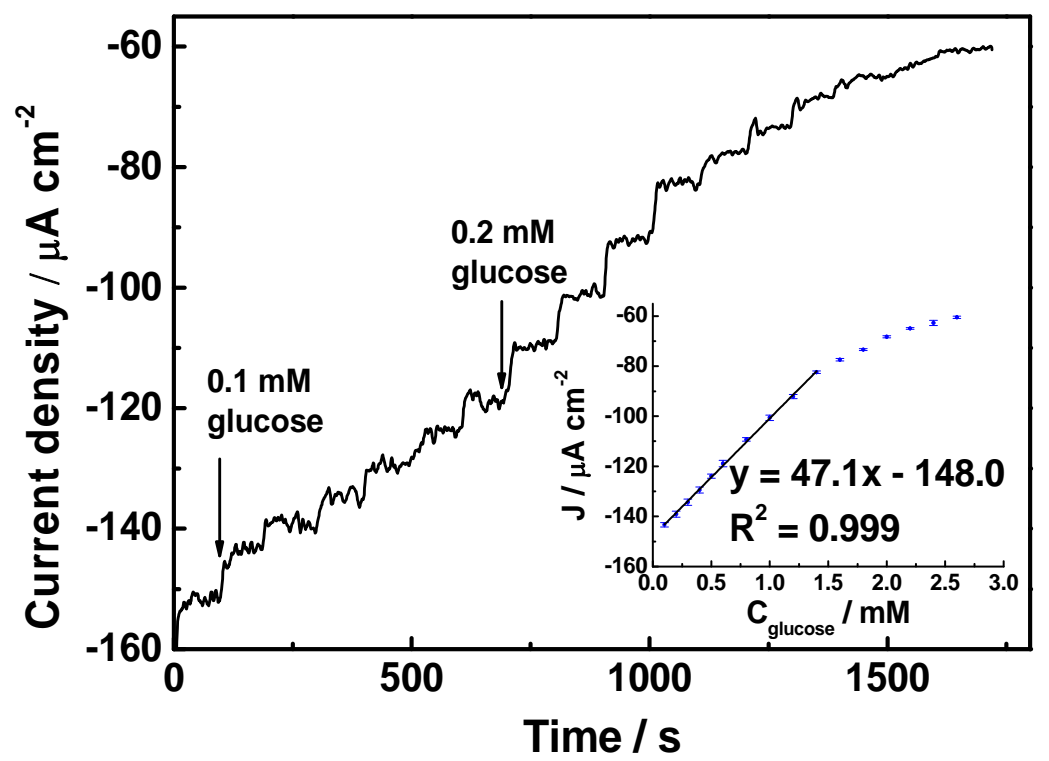

Fig. 4. Amperometric response of polyCo/GOx/GCE at a constant potential of $-0.8 \mathrm{~V}$ upon successive addition of glucose to the PBS electrolyte. Inset: calibration curve for average steadystate current density vs. glucose concentration. 


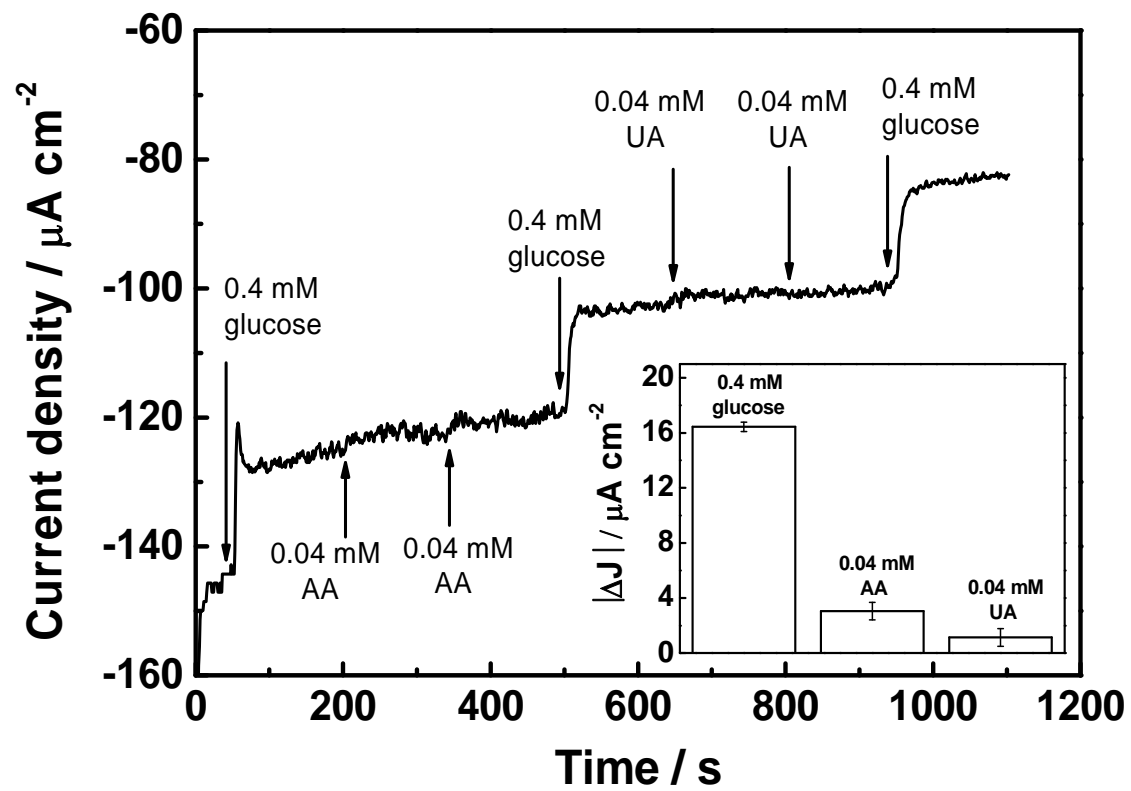

Fig. 5. Amperometric response of polyCo/GOx/GCE at a constant potential of $-0.8 \mathrm{~V}$ upon successive addition of $0.4 \mathrm{mM}$ glucose to the PBS electrolyte, with $0.04 \mathrm{mM} \mathrm{AA}$ and $0.04 \mathrm{mM}$ UA additions in between. Inset: the sensing selectivities of the biosensor. 


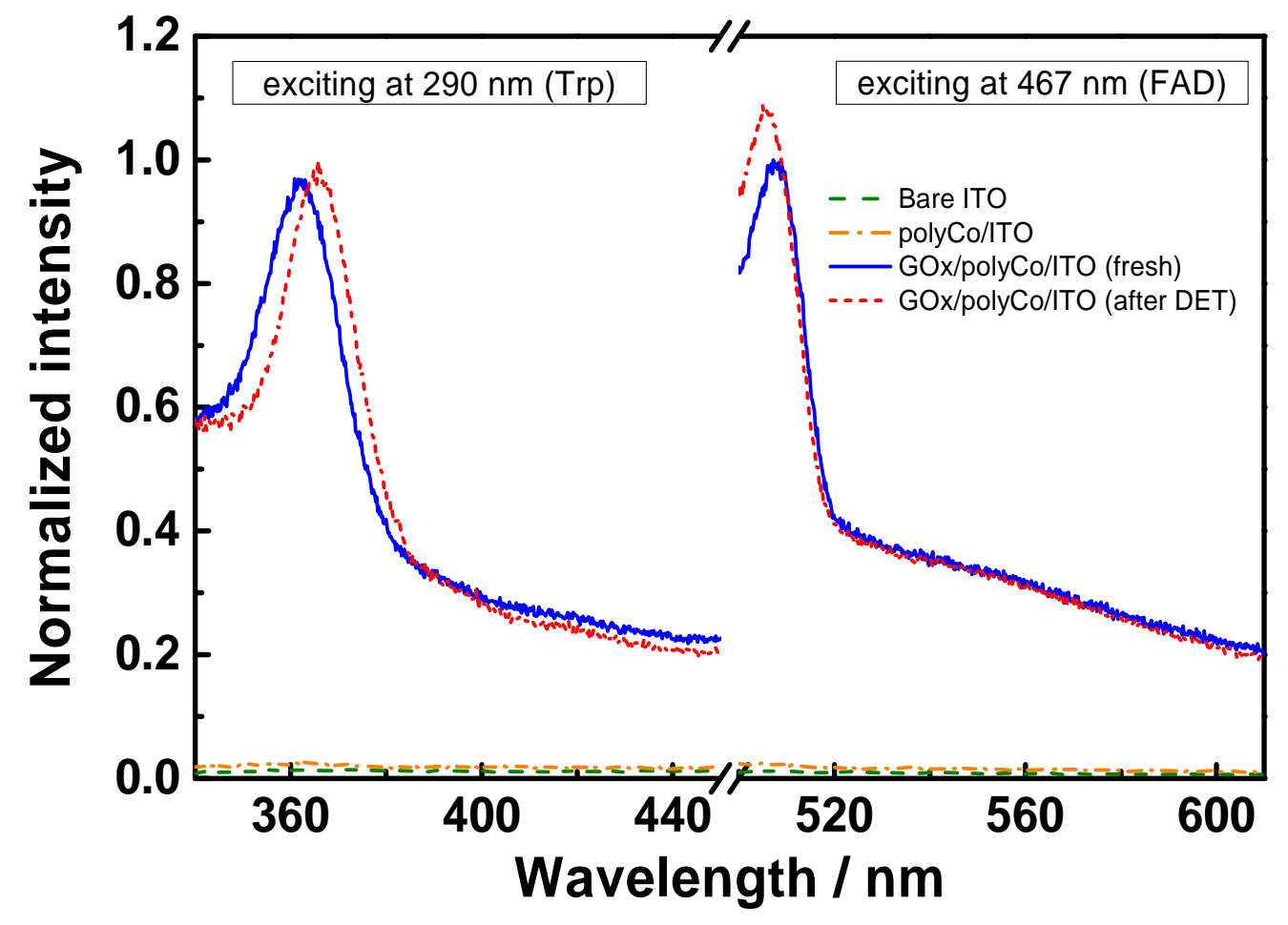

Fig. 6. Fluorescence spectra of bare ITO (green dashed line), polyCo/ITO (orange dash-dot line), and the GOx/polyCo/ITO freshly prepared film before (blue solid line) and after (red short dashed line) $100 \mathrm{CV}$ scans between 0 and $-1.2 \mathrm{~V}$ in PBS, excited at 290 or $467 \mathrm{~nm}$. All data were normalized based on the maximum intensity of the fresh GOx/polyCo/ITO at 366 or 508 $\mathrm{nm}$. The spectra of bare ITO (green dashed line) and polyCo/ITO (orange dash-dot line) are close to the baseline due to absence of emission. 
Table 1. Summary of the current changes for biosensor stability testing.

\begin{tabular}{cccc|ccc}
\hline $\boldsymbol{\Delta} \boldsymbol{J} / \boldsymbol{\mu} \mathbf{A ~ c m}^{-2}$ & Test 1 & Test 2 & Test 3 & Avg. & SD & RSD \\
\hline Fresh & 13.2 & 18.9 & 15.1 & 15.7 & 2.9 & $18.5 \%$ \\
Week 1 & 17.9 & 13.9 & 14.4 & 15.4 & 2.2 & $14.3 \%$ \\
Week 2 & 17.0 & 14.1 & 15.4 & 15.5 & 1.5 & $9.6 \%$ \\
Week 3 & 14.8 & 14.5 & 13.3 & 14.2 & 0.8 & $5.6 \%$ \\
Week 4 & 13.4 & 14.1 & 18.9 & 15.5 & 3.0 & $19.3 \%$ \\
\hline
\end{tabular}




\section{Graphical Abstract}

A Co(II)-Based Metallo-Supramolecular Polymer as a Novel Enzyme Immobilization Matrix for Electrochemical Glucose Biosensing

Chih-Yu Hsu, Takashi Sato, Satoshi Moriyama, and Masayoshi Higuchi
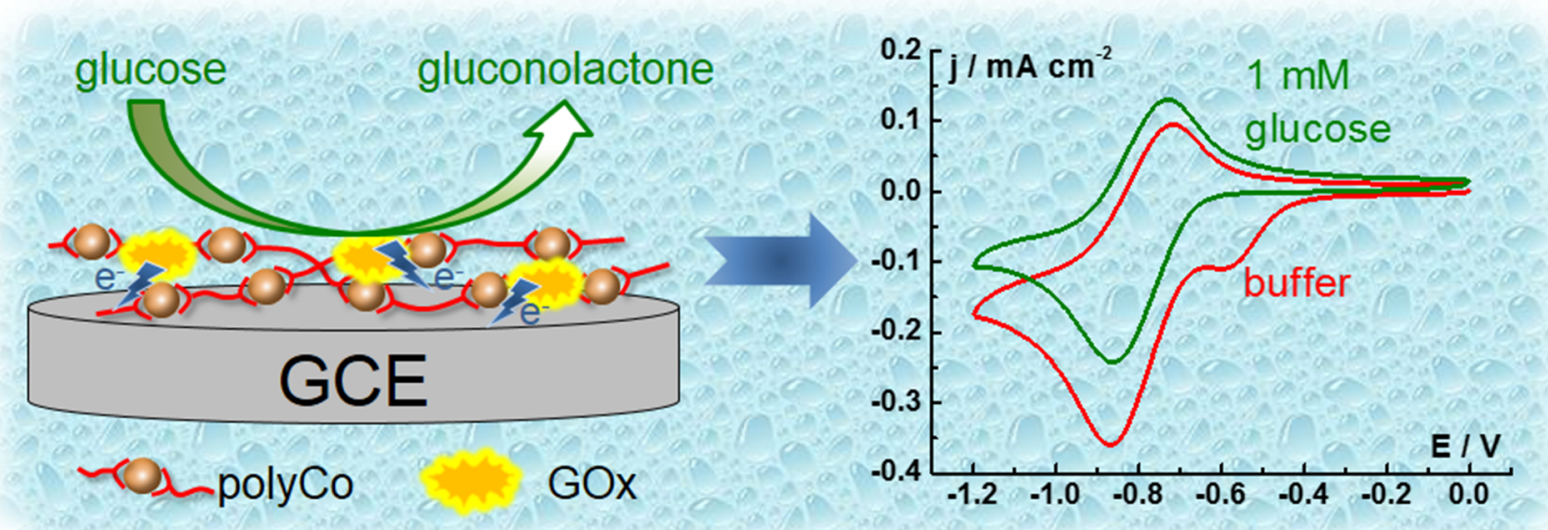